\title{
A new species of Ophryotrocha (Annelida: Dorvilleidae) associated with the coral Lophelia pertusa (Anthozoa: Caryophylliidae)
}

\author{
Vinicius da Rocha Miranda ${ }^{1,2}$; Andrielle Raposo Rodrigues ${ }^{1,3}$ \& Ana Claudia dos Santos Brasil ${ }^{1,4}$ \\ 1 Universidade Federal Rural do Rio de Janeiro (UFRRJ), Instituto de Ciências Biológicas e da Saúde (ICBS), \\ Departamento de Biologia Animal, Laboratório de Polychaeta. Seropédica, RJ, Brasil. \\ 2 ORCID: http://orcid.org/0000-0002-4591-184X.E-mail: vinicius.ghostty@gmail.com (corresponding author) \\ ${ }^{3}$ ORCID: http://orcid.org/0000-0001-9152-355X. E-mail: andriraposo@gmail.com \\ ${ }^{4}$ ORCID: http://orcid.org/0000-0002-0611-9948. E-mail: acbrasil@gmail.com
}

\begin{abstract}
Ophryotrocha is the most speciose genus within Dorvilleidae, with species occurring in a great variety of environments around the globe. In Brazil, records of Ophryotrocha are scarce and no specific identification is provided for any of the records. Herein we describe a new species of Dorvilleidae, Ophryotrocha zitae sp. nov. Adult and larval specimens were found in the axis of a fragment of the cold-water coral Lophelia pertusa, sampled off São Paulo's coast, at a depth of $245 \mathrm{~m}$. Both forms are described and illustrated. This new species resembles 0 . puerilis, 0 . adherens and 0 . eutrophila, but can be distinguished based on differences in its mandible and on chaetae shape and arrangement.
\end{abstract}

Key-Words. Epibiont; Cold-water Coral; Deep-sea; Eunicida, Associated fauna.

\section{INTRODUCTION}

The Family Dorvilleidae is comprised of 38 valid genera, many of which are monospecific (Read, 2016) and others, despite more specious, present evident morphological homogeny (Rouse \& Pleijel, 2001). Among them, Ophryotrocha is the genus with the largest number of valid species 72 living species and 2 fossil records (Read, 2016). The species of this genus occur from shallow to deep waters, being most abundant in environments rich in organic matter, as well as in sunken bones and in reducing environments (Paavo et al., 2000; Dahlgren et al., 2001; Rouse \& Pleijel, 2001; Paxton \& Morineaux, 2009; Wiklund et al., 2012; Taboada et al., 2013; Salvo et al., 2014). Many species are used as models for embryological, behavioral, evolutionary and bioassay studies due to their easy maintenance in the laboratory (Åkesson, 1970; Brown \& Ahsanullah, 1971; Pfannenstiel, 1977; Hooftman \& Vink, 1980; Sella, 1985, 1991; Sella et al., 1997; Paavo et al., 2000; Sella \& Lorenzi, 2000; Paxton, 2004; Prevedelli et al., 2006; Schleicherova et al., 2010).

External morphology is quite similar among species of the genus, being distinguished by the size of appendices such as antennae, palps and parapodial cirri; the presence of transverse ciliary bands on each segment; and the presence of ro- sette glands on the posterior region of the body (Ockelmann \& Åkesson, 1990; Heggoy et al., 2007; Paxton \& Åkesson, 2011). These species also bear a complex buccal apparatus comprising a pair of mandibles and maxillae, the latter being either "P-type" or "K-type", and the presence of one or two types of maxillae will vary according to each species (Paxton, 2004).

The reproductive modes occurring in Ophryotrocha have been thoroughly described for some of the species by Åkesson (1975) and Sella (2006). The group presents both hermaphroditic and gonochoric species; in both cases reproduction occurs in pairs and eggs are released in a cocoon, which can be guarded by one of the parents. Hatching time and larval size varies interspecifically and, after hatching, larvae start actively foraging around the cocoon (Paavo et al., 2000; Sella, 2006). Newly-released larvae resemble adults, differing from them by the presence of a third pygidial stylus. When the pygidial stylus is no longer visible, individuals can be considered young immature (Paavo et al., 2000).

Records of the genus in the South Atlantic are scarce, with a few records on the Argentinian coast and Falkland Islands (OBIS, 2019; Orensanz, 1973). For the Brazilian coast, to date, there is only one previous report of the genus Ophryotrocha - a non-identified specimen occurring on north- 
east region, found in shallow waters and associated with brown algae of the genus Dictyota (Cunha et al., 2013). Herein, we describe Ophryotrocha zitae sp. nov., which was sampled in association with the cold-water coral Lophelia pertusa (Linnaeus, 1758) off the north coast of São Paulo.

\section{MATERIAL AND METHODS}

The material analyzed in this work was obtained from colonies of Lophelia pertusa sampled in Santos Basin $\left(24^{\circ} 21^{\prime} 27,19^{\prime \prime} \mathrm{S} ; 44^{\circ} 13^{\prime} 20,95^{\prime \prime} \mathrm{W}\right), 136 \mathrm{~km}$ off the north coast of São Paulo, Brazil. Random samples of coral were acquired from this area by the research and development center of the Brazilian energy company Petrobras (CENPES). Polychaetes were manually removed from a fragment of $L$. pertusa, fixed in formalin and stored in $70 \% \mathrm{EtOH}$.

Parapodia were mounted on permanent slides using Gray \& Wess (PVA) (Humason, 1979 ) and Hoyer's mounting media (Krantz, 1978). To observe jaws and mandibles, four adult specimens were clarified in a solution of $10 \%$ $\mathrm{KOH}$, before being mounted on a permanent slide using Hoyer's mounting media. Description of these latter structures followed Paxton (2004). Specimens submitted to scanning electron microscopy (SEM) were dehydrated following the procedures described by Fitzhugh \& Rouse (1999). Briefly, specimens were initially dehydrated through a graded ethanol (EtOH) series, then the EtOH was replaced by Hexamethyldisilazane (HMDS) through a graded series, and finally the HMDS was evaporated off. After this procedure, the specimens were covered with gold-palladium and examined in a JEOL JSM-6390LV at the Museu Nacional of the Universidade Federal do Rio de Janeiro. The measurements were made on fixed specimens; the length encompasses the anterior-most portion of the prostomium to the posterior-most portion of the pygidial segment (excluding cirrostyles); width was measured on a median segment, including parapodial lobe.

All drawings were made with the aid of a camera lucida and photographs were taken using an adapted Sony Cybershot W350. All specimens are deposited in the Polychaeta collection located at the Museu Nacional of the Universidade Federal do Rio de Janeiro (MN-UFRJ).

\section{SYSTEMATICS}

Family DORVILLEIDAE Chamberlin, 1919 Genus Ophryotrocha Claperède \& Metschnikow, 1869 Ophryotrocha zitae sp. nov. (Figs. 1-2)

Type material: Holotype: MNRJP2601: 1 specimen

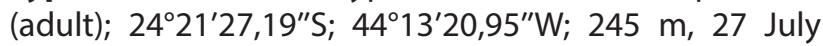
2010; on Lophelia pertusa. Paratypes: MNRJP2602:

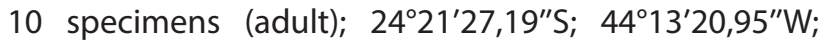
245 m, 27 July 2010; on Lophelia pertusa. MNRJP2603:
4 specimens (3 juveniles and 1 larva); $24^{\circ} 21^{\prime} 27,19^{\prime \prime} S$; $44^{\circ} 13^{\prime} 20,95^{\prime \prime} \mathrm{W} ; 245$ m, 27 July 2010; on Lophelia pertusa.

Non-type material: MNRJP2604: 59 specimens; $24^{\circ} 21^{\prime} 27,19^{\prime \prime}$ S; $44^{\circ} 13^{\prime} 20,95^{\prime \prime} \mathrm{W} ; 245$ m, 27 July 2010; on Lophelia pertusa.

Diagnosis: K-maxillae comprising a heavily-sclerotized main fang and seven partially fused plates; P-maxillae consisting of seven rounded plates, two of them fused and five only partially fused. Uniramous parapodia with a bundle of capillary chaetae occurring only on the supra-acicular region. Sub-acicular region possessing a bundle of heterogomph falcigers with slightly serrated blades and single capillary chaeta. Rosette glands from segment eleven on.

\section{DESCRIPTION}

Measurements: Holotype: $3.5 \mathrm{~mm}$ long, $0.8 \mathrm{~mm}$ wide; Paratypes: mean size: $1.8 \mathrm{~mm}$ long, $0.5 \mathrm{~mm}$ wide (largest specimen $4.20 \mathrm{~mm}$ long, $0.9 \mathrm{~mm}$ wide; smallest specimen $0.65 \mathrm{~mm}$ long, 0,22 $\mathrm{mm}$ wide).

Body short (holotype with 24 segments), stout, dorsally convex, ventral region flattened, with longitudinal groove. Both ends are terminally rounded (Figs. 1A-B). Bundles of cilia present throughout body. On the prostomium these form a frontal bundle with tufts between the antennae and the eyes (Figs. $1 \mathrm{~A}-\mathrm{C}$ ), while complete rings of cilia occur on each peristomial segment and on the pygidium, whereas remaining segments possess a dorsal and a ventral bundle of cilia separated by the parapodia (Figs. 1A-B, for dorsal bundle). Fixed specimens with an opaque pale white color (Fig. 1B).

Prostomium wider than longer (Figs. 1A-C). Anterior border with up to 10 tactile cilia. Cirriform antennae inserted dorsolaterally, with a tuft of stereocilia on the apex (Fig. 1C). Palps digitiform, stout, inserted laterally on the prostomium (Fig. 1C). A pair of eyes is located posteriorly, near the edge of the first peristomial segment, eyes exhibiting a silver coloration under oblique illumination (Fig. 1B). Nuchal organs consisting of a pair of ciliate tufts located just after and between the eyes. Peristomial segments achaetous, with a translucid region on the dorsum from which it is possible to observe the buccal apparatus (mandible and maxillae) (Figs. 1A-C). Mouth large, aperture half the size of segment width, located anteriorly on the first segment.

The K-maxillae are fully developed, composed of two black to dark brown forceps with single tips, united at the posterior end (Figs. 1D, 2A). The light brown carrier-like structure is connected to the forceps by narrow ligaments - with basal lateral sheets - that arise from the posterior half of the forceps and connect to the first of the articulated denticles (Figs. 1D, 2A). This carrier-like structure is composed of seven spoon-like denticles, which become larger from the first to the last; the cutting edges of the first to third denticles are coarsely serrated, with the teeth alternating in size (the larger ones about 
twice the size of the smaller ones), while the cutting edges of the fourth to seventh denticles are finely serrated with all teeth of similar size (Fig. 2A).

Mandibles are elongated sclerotized shafts, dark brown in color at the center and becoming translucent towards the edges, located ventrally to the maxillae (Figs. 1D, 2B). Anterior edges of mandibles representing the cutting plates with about 20 very small and worn teeth; apophysis emerging postero-ventrally to the cutting plate, three times the width of same at the largest region and becoming narrow posteriorly; shafts longer than wide with posterior apex ending on a bend towards the middle of the body; light brown internal lateral projections present subdistally.
Chaetigerous segments without lobes protruding towards parapodia. Rosette glands occurring from the eleventh segment - in which they occur only on the right side - to the end of the body, where they are so small that they are difficult to observe without staining (Fig. 1A). Body tapering posteriorly. Pygidium bearing a pair of ventral cirri, pygidial stylus absent. Anus terminal and located dorsal to the cirri (Figs. 1A-B).

Parapodia uniramous, with triangular (on anterior segments) to rounded (on posterior segments) pre-chaetal lobes longer than post-chaetal ones, with both dorsal and ventral smooth cirri present (Fig. 2C); retractile lobes on the posterior side of parapodia and almost imperceptible in the specimens, supported by a single smooth

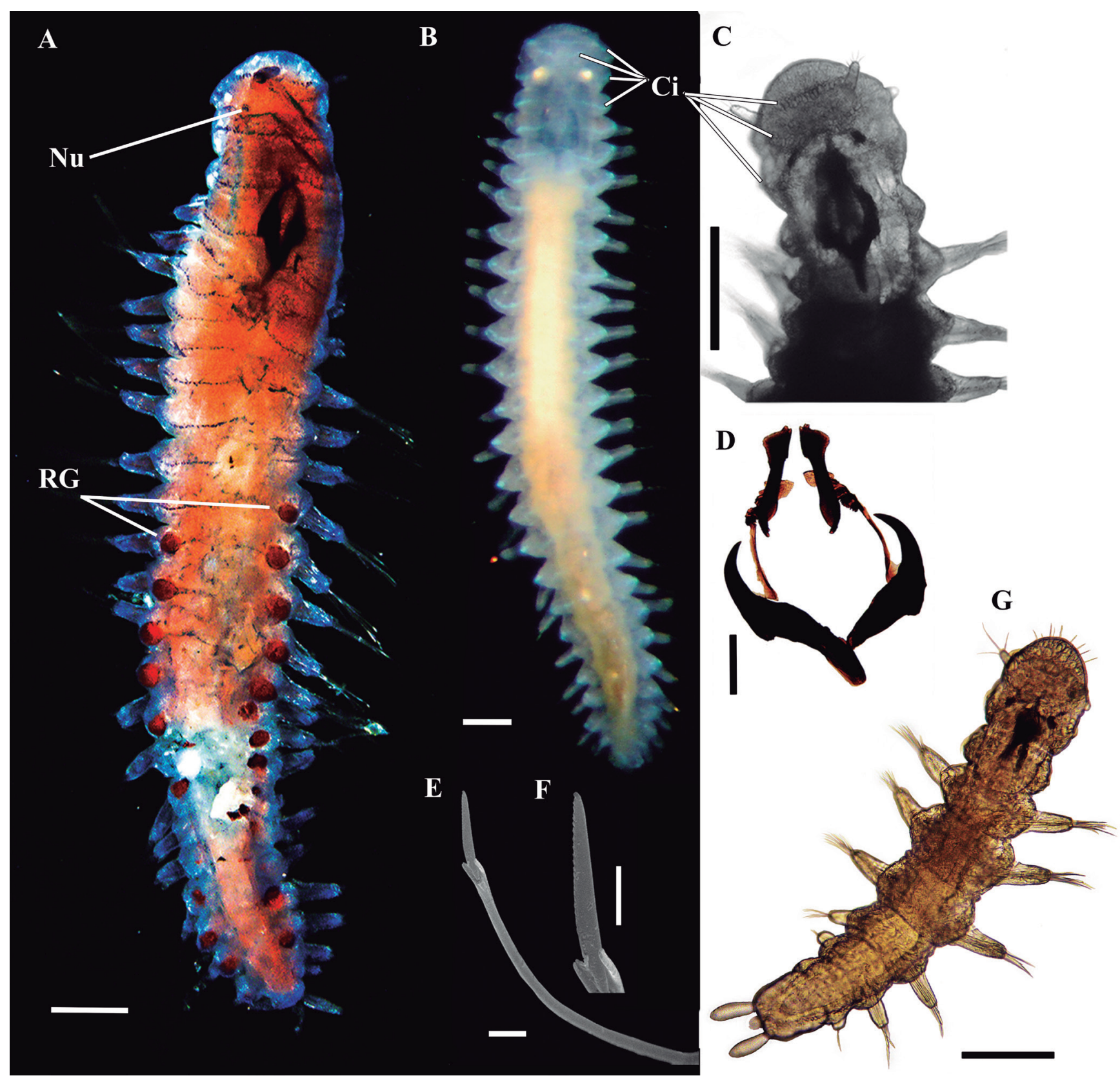

Figure 1. Ophryotrocha zitae sp. nov.: (A) Holotype (MNRJP2601), dorsal view, stained with Shirlastain; (B) Paratype (MNRJP2602), dorsal view, unstained; (C) Prostomium of a Paratype (MNRJP2602); (D) Buccal apparatus; (E) SEM image of a compound neurochaetae from a middle segment; (F) Detail of blade from the same; (G) Juvenile paratype (MNRJP2603), dorsal view. Abbreviations: $\mathrm{Ci}=$ Ciliary bands, $\mathrm{Nu}=$ Nuchal organ, $\mathrm{RG}=$ Rosette glands. $\mathrm{Scale}$ bars: $\mathrm{A}-\mathrm{C}=0,5 \mathrm{~mm}$; $D=250 \mu \mathrm{m} ; E-F=100 \mu \mathrm{m} ; G=01 \mathrm{~mm}$. 


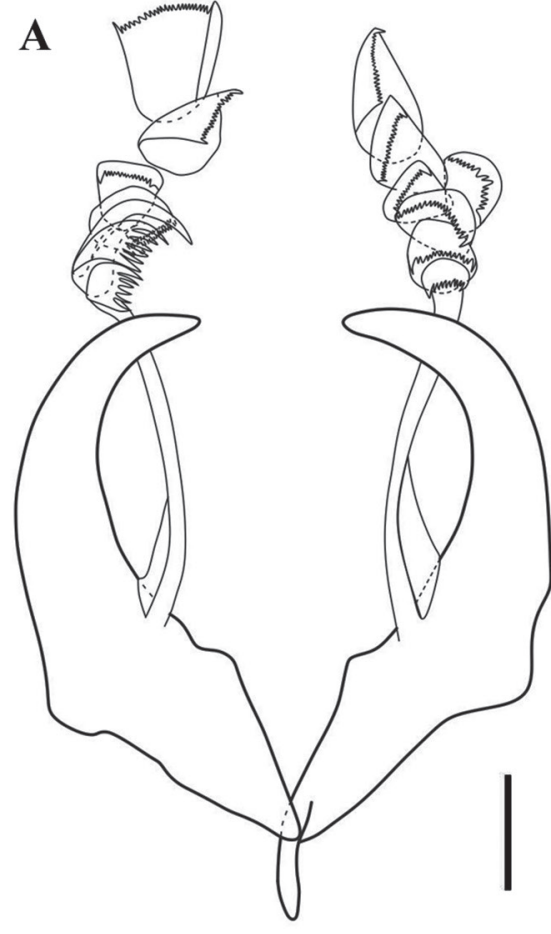

$\mathbf{C}$

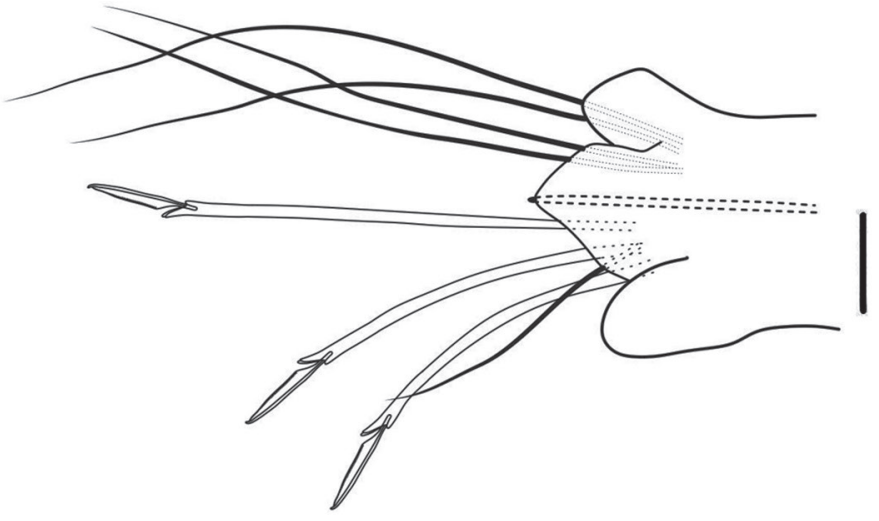

H
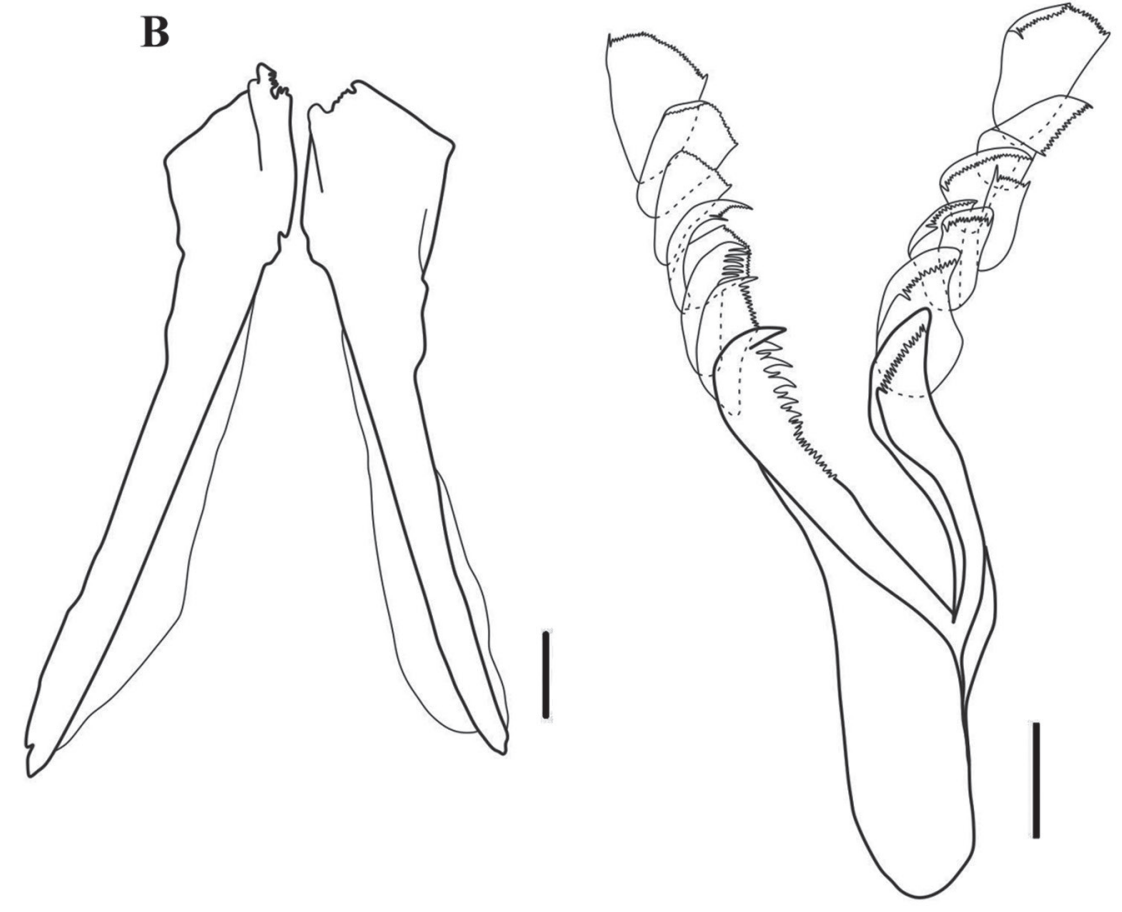

C



G

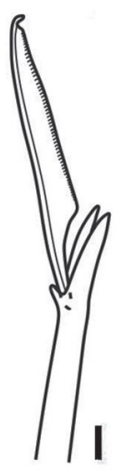

I
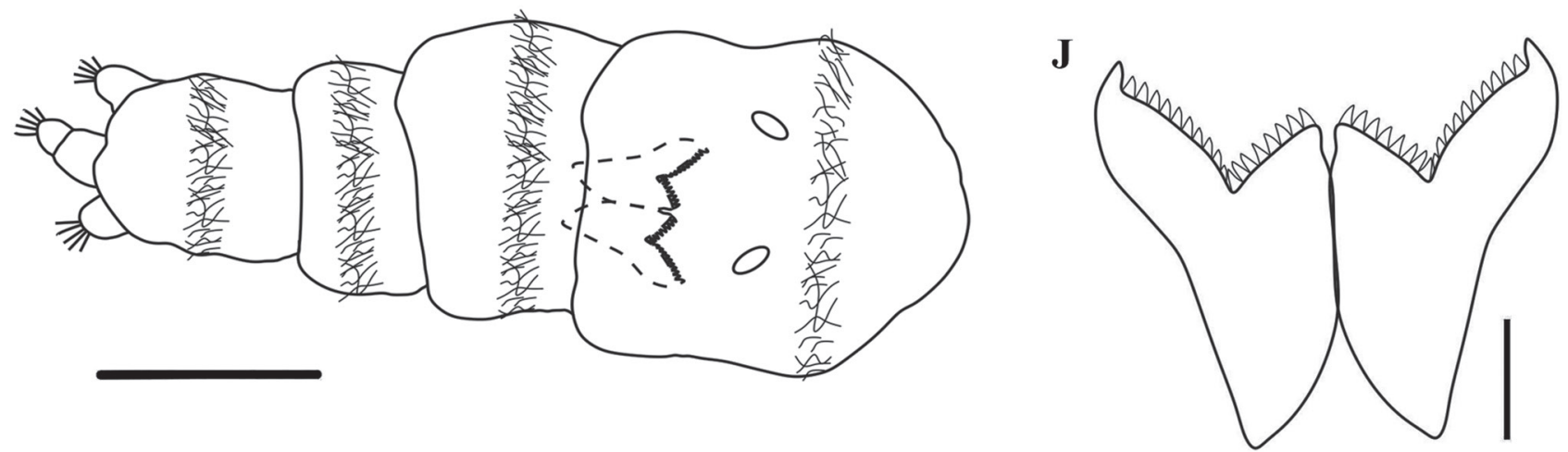

Figure 2. Ophryotrocha zitae sp. nov.: (A) K-type maxilla; (B) Adult mandible; (C) Parapodia from a middle segment; (D) Blade from upper compound chaeta from the first chaetiger; (E) Blade from lower compound chaeta from the first chaetiger; (F) Blade from upper compound chaeta from a middle chaetiger; (G) Blade from lower compound chaeta from a middle chaetiger; (H) P-type maxilla; (I) Larva paratype (MNRJP2603), dorsal view; (J) Mandible from larva. Scale bars: A = 250 $\mu$ m; $B=175 \mu \mathrm{m} ; C=500 \mu \mathrm{m} ; D-H=200 \mu \mathrm{m} ; \mathrm{I}=600 \mu \mathrm{m} ; \mathrm{J}=120 \mu \mathrm{m}$. 


\begin{tabular}{|c|c|c|c|c|c|c|c|c|c|c|c|c|c|c|c|}
\hline 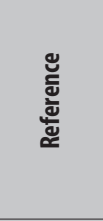 & 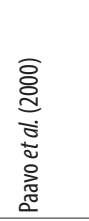 & 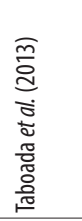 & 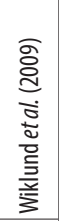 & 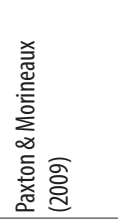 & 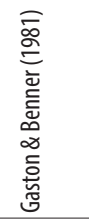 & 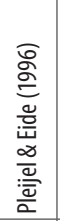 & 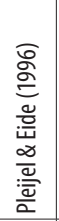 & 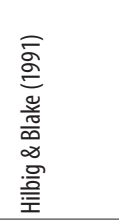 & 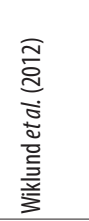 & 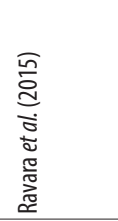 & 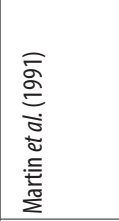 & 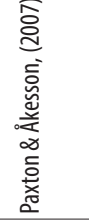 & 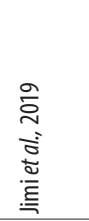 & 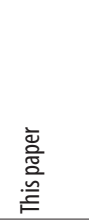 & 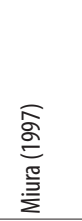 \\
\hline 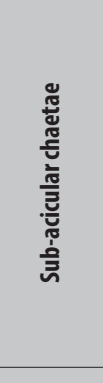 & 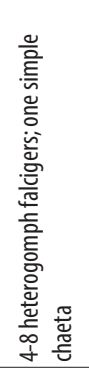 & 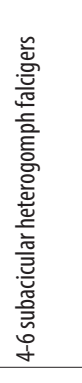 & 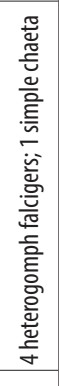 & 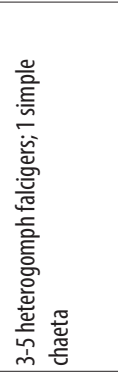 & 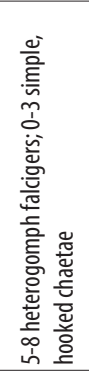 & $\sim$ & $\sim$ & 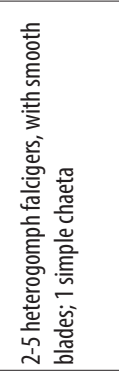 & 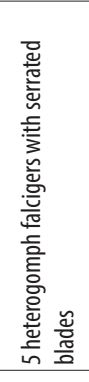 & 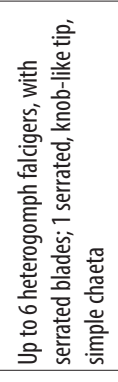 & 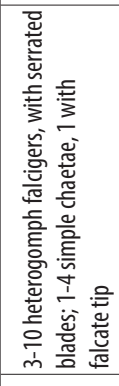 & 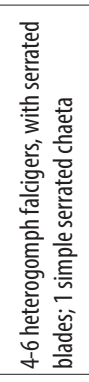 & 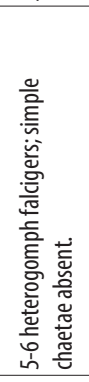 & 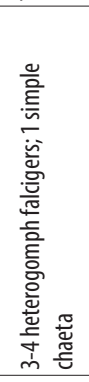 & 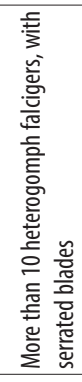 \\
\hline 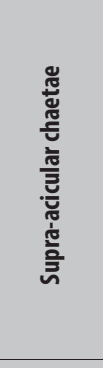 & 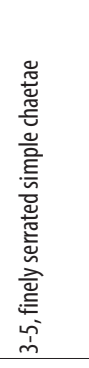 & 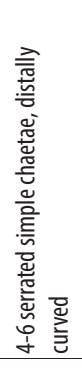 & 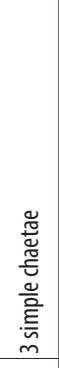 & 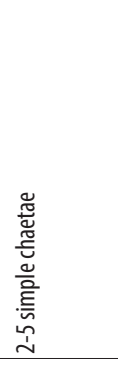 & 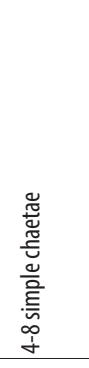 & $\sim$ & $\sim$ & 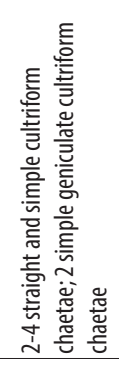 & 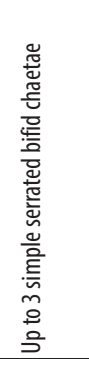 &  & 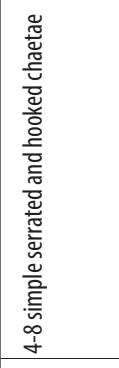 & 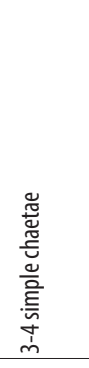 & 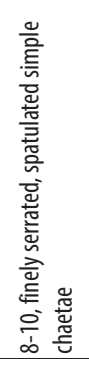 & 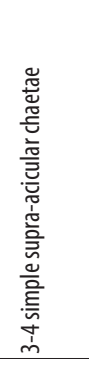 & 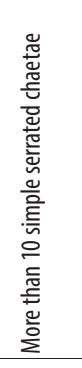 \\
\hline 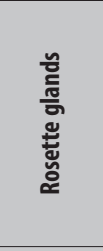 & $\sim$ & $\sim$ & $\sim$ & $\sim$ & $\sim$ & 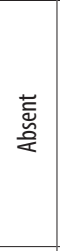 & $\begin{array}{l}\text { 莺 } \\
\text { 晏 }\end{array}$ & $\sim$ & $\sim$ & 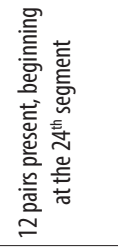 & $\sim$ & 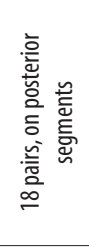 & $\sim$ & 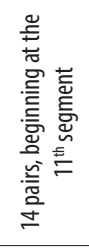 & $\sim$ \\
\hline$\frac{\text { 응 }}{\frac{\pi}{2}}$ & 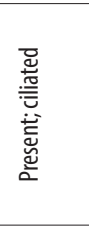 & $\begin{array}{l}\text { 志 } \\
\text { 衷 }\end{array}$ & 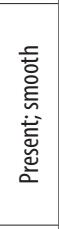 &  & 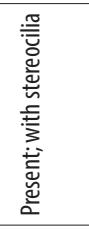 & 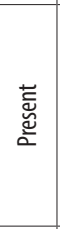 & $\begin{array}{l}\text { 志 } \\
\text { 产 }\end{array}$ & $\begin{array}{l}\text { 咅 } \\
\text { 宸 }\end{array}$ & 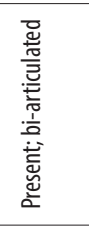 & 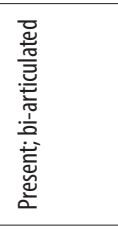 & 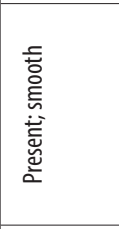 & 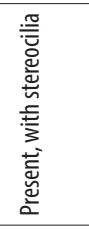 & 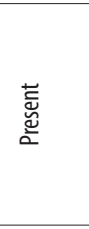 & 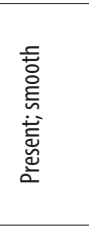 & 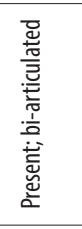 \\
\hline 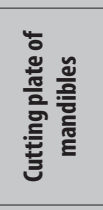 & 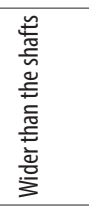 & 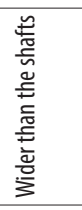 &  & 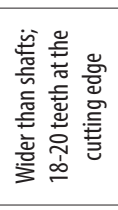 & 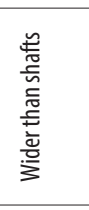 & $\sim$ & $\sim$ & 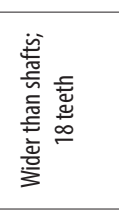 & 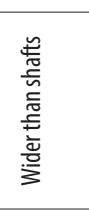 &  & 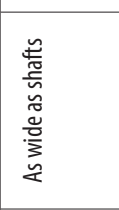 & 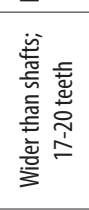 & 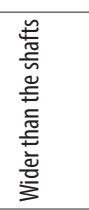 & 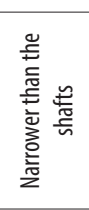 & 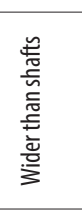 \\
\hline 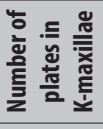 & 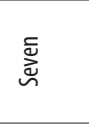 & 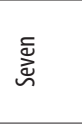 & 产 & 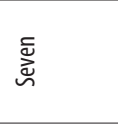 & $\underset{\sim}{x}$ & 尝 & 壹 & 言 & ڤ్ & $\approx$ & 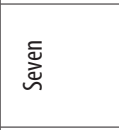 & 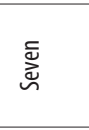 & 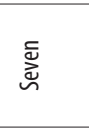 & 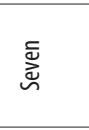 & 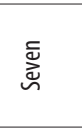 \\
\hline$\stackrel{\tilde{y}}{\tilde{y}}$ & 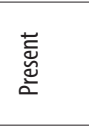 & 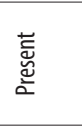 & 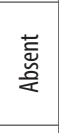 & 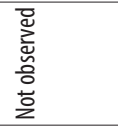 &  & 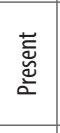 & $\begin{array}{l}\text { 莺 } \\
\text { 旁 }\end{array}$ & $\begin{array}{l}\text { 䓌 } \\
\text { 寊 }\end{array}$ & 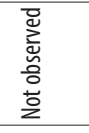 & $\begin{array}{l}\text { 䓌 } \\
\text { 衷 }\end{array}$ & 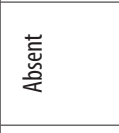 & 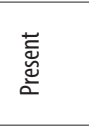 & 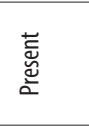 & 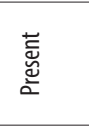 & $\sim$ \\
\hline 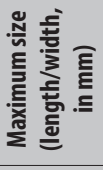 & $\tilde{F}$ & $\begin{array}{l}\stackrel{0}{0} \\
\stackrel{\sigma}{0}\end{array}$ & $\tilde{\infty}$ & ్ㅗㅇ & 孰 & $\lesssim$ & $\approx$ & 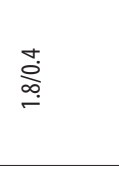 & 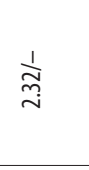 & $\tilde{\vec{j}}$ & 岕 & 今ે & $\stackrel{n}{\stackrel{n}{N}}$ & 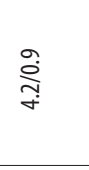 & 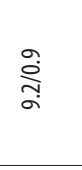 \\
\hline 屁 & 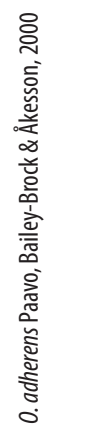 & 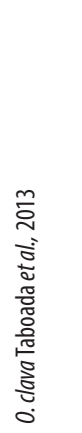 & 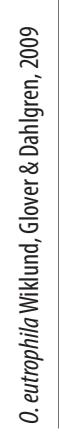 &  & 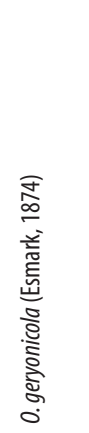 & 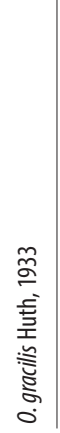 & 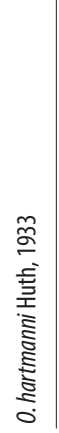 & 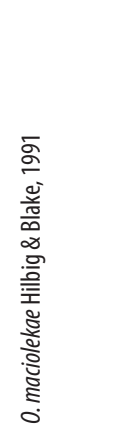 & 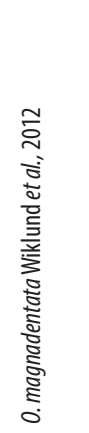 & 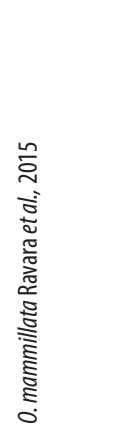 & 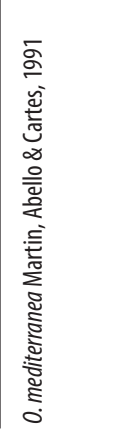 &  &  & 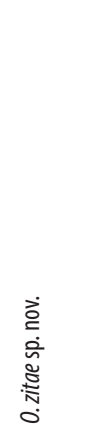 & 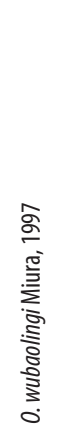 \\
\hline
\end{tabular}


chaeta. Three groups of chaetae emerge from the parapodia: a bundle of three to four simple chaetae supra-acicular; and another bundle of three to four heterogomph falcigers emerging right below the acicula (Fig. 2C); below the bundle of compound chaetae there is a single simple chaetae, similar to those of the supra-acicular bundle. The shafts of compound chaetae smooth (Fig. 1E); internal border of the blades slightly serrated and presenting a small terminal concavity (Figs. 1E-F, 2D-G), which becomes more prominent on posterior segments. Upper compound chaetae (Figs. 2D and F) with blades longer than those below (Figs. 2E and G), also the blades in the chaetae of the anterior parapodia (Figs. 2D-E) are longer than those in the posterior parapodia (Figs. 2F-G).

Juvenile: Specimens resembling adults, with six chaetigers (Fig. 1G). Prostomium rounded and wider than long, with a tuft of cilia at the anterior border and another tuft - forming a complete ring around the prostomium - posterior to this and anterior to the eyes. Antennae not observed. A pair of digitiform palps emerging laterally to the prostomium, each with a tuft of three stereocilia at the apex. A pair of light brown eyes situated on the posterior half of the prostomium. Nuchal organs present posteriorly to the eyes. Following the prostomium are two achaetous segments, each with a tuft of cilia forming a complete ring around them.

Mouth located at the anterior border of the first peristomial segment. Mandibles are like those in the holotype. Maxillae of P2 type, with a dark amber color. Forceps with a curved distal fang double the size of other teeth; anterior half coarsely serrated; posteriorly, the forceps are fused forming a shaft (Fig. 2H). Carrier-like structure composed of seven pairs of partially articulated denticles, the first three pairs of which are coarsely serrated, and the other four pairs finely serrated and with a major fang on the external margin of the serrated border.

Chaetigerous segments as described for the holotype. Pygidium with two long smooth cirri, one on either side of the terminal anus. A bulbous structure (a remaining of pygidial stylus) is present dorsally to the anus (Fig. 1G).

Larva: Among the specimens analyzed, a single larva was found. The specimen has only three achaetous segments (3.01 $\mu \mathrm{m}$ long; $0.5 \mu \mathrm{m}$ wide) (Fig. 2l). The prostomium is rounded and without palps or antennae but with a pair of dark eyes located posteriorly; two ciliary tufts circle the prostomium (one near the anterior border and another at the middle region) (Fig. 2I). A pair of Y-like mandibles located on the first segment; cutting edges with 20 prominent teeth (the second external-most one twice the size of the remaining teeth and this feature was observed only in the larva); teeth located internally curved towards the external margin while those located externally are curved towards the interior; shafts small with narrow lateral expansions (Fig. 2J). P1-maxillae present, amber to brown in color and composed of four pairs of plates; first pair longer than the others and with their internal borders coarsely serrated (alternating larger and smaller teeth); remaining plates ovate (in the observed specimen, the second left plate is only half the size of the one on the right), internal borders serrated (alternating larger and smaller teeth on second and third plates, but teeth all the same size on the fourth plate). Remaining segments apodous, with two to three capillary chaetae emerging directly from the body wall; ciliary tufts completely encircling the segments (Fig. 2l). Pygidium bearing a pair of terminal pygidial cirrus and a dorsal pygidial stylus - the stylus having an annulation medially and double the size of the cirri - both located anteriorly to the terminal anus and both bearing a tuft of stereocilia at the apex (Fig. 2l).

Etymology: The species name is a tribute to the grandmother of the author A.C.S. Brasil, Maria Teresa dos Santos (known as Dona Zita): a strong woman, who exhibited the same devoted care to her descendants as seen for many species of this genus.

Habitat: The specimens were found in a depth of $245 \mathrm{~m}$, in association with the cold water coral Lophelia pertusa.

Type locality: Southwest Atlantic, Santos Basin, off the state of São Paulo, Brazil.

Remarks: The 75 currently-assigned species to the genus Ophryotrocha can be divided into three groups according to the morphology of the maxillae in adults: one encompassing the species in which adults bear P-maxillae, another in which the adults present K-maxillae with both fangs of the forceps as single tips, and a last group that also bear K-maxillae but with at least one of the fangs being bidentate. Based on this categorization, O. zitae sp. nov., falls into the group in which both fangs are unidentate. The 20 species of the second group of Ophryotrocha that have unidentate forceps are partially compared in Table 1, and those that most resemble O. zitae sp. nov., are: O. adherens Paavo, Bailey-Brock \& Åkesson, 2000, O. eutrophila Wiklund et al., 2009, and O. puerilis Claperède \& Metschnikow, 1869.

The morphology and ornamentation of the palps are distinguishing characters among $O$. zitae and the other three species. In O. zitae the palps are digitate and smooth, while $O$. adherens and $O$. puerilis have ovate palps with a tuft of cilia at the apex, and O. eutrophila have digitate palps.

Nuchal organs appear in different numbers among O. zitae, O. adherens and O. puerilis. The former has only a pair of nuchal organs located posteriorly on prostomium, while the other two species possess two pairs of nuchal organs.

The third pygidial stylus is a character described as present only on juveniles of Ophryotrocha, while adults lacks this structure (Paavo et al., 2000). However O. adherens, $O$. puerilis and $O$. eutrophila are all described as possessing a third stylus, while all mature specimens of $O$. zitae sp. nov. observed do not possess it. The occurrence of such structure in mature specimens and among the other species of Ophryotrocha needs to be verified, 
in order to confirm the statement of Paavo et al. (2000). Herein we assume that the presence of the third pigidial stylus in mature specimens is a distinguishing character among species of the genus.

In addition to the characters compared in Table 1, $O$. adherens differs from $O$. zitae sp. nov., by having an acicula that may emerge from within the flesh, and by the bifurcation at the tip of the supra-acicular chaetae. In 0 . adherens some of the compound chaetae may occur in the ventral setal lobe (which does not occur in O. zitae sp. nov.).

In relation to 0 . puerilis, we can point out the presence of tactile cilia at the anterior border of the prostomium of this species, while cilia at this region is absent in 0 . zitae sp. nov. Also, the absence of a tuft of cilia on the apex of the dorsal and ventral parapodial cirri in O. zitae sp. nov., in comparison with the presence of such ciliation in O. puerilis. The number of prostomial ciliary bands differ between both species: $O$. zitae sp. nov., has only a single band of cilia, while $O$. puerilis has two bands of cilia.

In relation to $O$. eutrophila the absence of eyes is a distinguishing character, while $O$. zitae sp. nov., possesses eyes. Parapodial cirri and retractile lobe contrast between both species, in O. eutrophila the cirri (dorsal and ventral) are smaller, while the retractile lobe is longer, in comparison with the same structures in O. zitae sp. nov. Morphology of the teeth in the P-type maxilla also differ between both species: the teeth in the cutting border of the D1 and D2 of O. eutrophila are longer than those in O. zitae sp. nov., also the D3 to D7 plates in O. eutrophila are longer and wider than in O. zitae sp. nov.

Species of Ophryotrocha have been described worldwide, but there are only three records of the genus from the South Atlantic: a non-identified species from northeastern Brazil (Cunha et al., 2013), and O. claparedei Studer, 1878 and O. notialis (Ehlers, 1908) both from Argentina (Orensanz, 1973, 1990), none of them resembling $O$. zitae sp. nov. While both Argentinian species have P-type maxillae only, the species described herein changes the P-type maxilla for a K-type along its development. Orensanz (pers. comm.) also identified some specimens of $O$. puerilis from an aquarium at Mar del Plata (Northern Argentina), but according to him the specimens present some differences from the original description provided by Claperède \& Metschnikow (1869), so until these specimens are re-examined we prefer to avoid comparisons.

\section{ACKNOWLEDGEMNTS}

We are grateful to Marcia Reynier from Labtox for sending to us the specimens described in this manuscript. Also, to Cenpes/Petrobras for helping Brazilian scientists to discover and describe the diversity of the continental margin. We also are grateful to all reviewers that made comments that improved the quality of the manuscript. The first author also would like to thank Coordenadoria de Aperfeiçoamento de Pessoal de Ensino Superior (CAPES) for his scholarship.

\section{REFERENCES}

Åkesson, B. 1970. Ophryotrocha labronica as test animal for the study of marine pollution. Helgoländer Wissenschaftliche Meeresuntersuchungen, 20: 293-303.

Åkesson, B. 1975. Reproduction in the genus Ophyotrocha (Polychaeta, Dorvilleidae). Pubblicazioni della Stazione Zoologica di Napoli, 39(Suppl. 1): 377-398.

Brown, B. \& Ahsanullah, M. 1971. Effect of heavy metals on mortality and growth. Marine Pollution Bulletin, 2: 182-187.

Chamberlin, R.V. 1919. The Annelida Polychaeta [Albatross Expeditions]. Memoirs of the Museum of Comparative Zoology at Harvard College, 48: $1-514$.

Claperède, É. \& Metschnikow, E. 1869. Beitraege zur Kenntnis der Entwicklungsgeschichte der Chaetopoden. Zeitschrift für Wissenschaftliche Zoologie, 19: 163-205.

Cunha, T.J.; Güth, A.Z.; Bromberg, S. \& Sumida, P.Y.G. 2013. Macrofauna associated with the brown algae Dictyota spp. (Phaeophyceae, Dictyotaceae) in the Sebastião Gomes Reef and Abrolhos Archipelago, Bahia, Brazil. Continental Shelf Research, 70: 140-149.

Dahlgren, T.G.; Åkesson, B.; Schander, C.; Halanych, K.M. \& Sundberg, P. 2001. Molecular phylogeny of the model annelid Ophryotrocha. Biological Bulletin, 201(2): 193-203.

Fitzhugh, K. \& Rouse, G.W. 1999. A remarkable new genus and species of fan worm (Polychaeta: Sabellidae: Sabellinae) associated with marine gastropods. Invertebrate Biology, 118(4): 357-390.

Gaston, G.R. \& Benner, D.A. 1981. On Dorvilleidae and Iphitimidae (Annelida: Polychaeta) with a redescription of Eteonopsis geryonicola and a new host record. Proceedings of the Biological Society of Washington, 94: 76-87.

Heggoy, K.K.; Schander, C. \& Åkesson, B. 2007. The phylogeny of the annelid genus Ophryotrocha (Dorvilleidae). Marine Biology Research, 3: 412-420.

Hilbig, B. \& James, A. 1991. Dorvilleidae (Annelida: Polychaeta) from the U.S. Atlantic slope and rise. Description of two new genera and 14 new species, with generic revision of Ophryotrocha. Zoologica Scripta, 20(2): 147-183.

Hooftman, R.N. \& Vink, G.J. 1980. The determination of toxic effects of pollutants with the marine polychaete worm Ophryotrocha diadema. Ecotoxicology and environmental safety, 4: 252-262.

Humason, G.L. 1979. Animal tissue techniques. 4. ed. San Francisco, Freeman and Company.

Jimi, N.; Taru, M. \& Imura, S. 2019. Life in the city: a new scavenger species of Ophryotrocha. Proceedings of the Biological Society of Washington, 132: 131-140.

Krantz, G. 1978. A manual of acarology: Field Guide and Catalogues. Corvallis, Oregon State University Book Stores. 509p.

Linnaeus, C. 1758. Systema Naturae per Regna Tria Naturae, Secundum Classes, Ordines, Genera, Species, Cum Characteribus, Differentiis, Synonymis, Locis. 10. ed. Holmiae, Laurentius Salvius.

Martin, D.; Abelló, P. \& Cartes, J. 1991. A new species of Ophryotrocha (Polychaeta: Dorvilleidae) commensal in Geryon longipes (Crustacea: Brachyura) from the Western Mediterranean Sea. Journal of Natural History, 25: 279-292.

Miura, T. 1997. Two new species of the genus Ophryotrocha (Polychaeta, Iphitimidae) from Kagoshima Bay. Bulletin of Marine Science, 60: 300-305.

Ocean Biogeographic Information System (OBIS). 2019. Ocean Biogeographic Information System: Ophryotrocha Claparède \& Mecznikow, 1869. Available at: https://obis.org/taxon/129266. Access in: 30/12/2019.

Ockelmann, K.W. \& Åkesson, B. 1990. Ophryotrocha socialis n. sp., a link between two groups of simultaneous hermaphrodites within the genus (Polychaeta, Dorvilleidae). Ophelia, 31: 145-162. 
Orensanz, J.M. 1973. Los anelidos poliquetos de la provincia biogeografica Argentina. III. Dorvilleidae. Physis, Sec A, 32(85): 325-342.

Orensanz, J.M. 1990. The Eunicemorph polychaete annelids from Antarctic and Subantarctic seas. Antarctic Research Series, 52: 1-183.

Paavo, B.; Bailey-Brock, J.H. \& Åkesson, B. 2000. Morphology and life history of Ophryotrocha adherens sp. nov. (Polychaeta, Dorvilleidae). Sarsia, 85: 251-264.

Paxton, H. 2004. Jaw growth and replacement in Ophryotrocha labronica (Polychaeta, Dorvilleidae). Zoomorphology, 123: 147-154.

Paxton, H. \& Åkesson, B. 2007. Redescription of Ophryotrocha puerilis and 0. Iabronica (Annelida, Dorvilleidae). Marine Biology Research, 3: 3-19.

Paxton, H. \& Åkesson, B. 2011. The Ophryotrocha diadema group (Annelida: Dorvilleidae), with the description of two new species. Zootaxa, 3092: 43-59.

Paxton, H. \& Morineaux, M. 2009. Three species of Dorvilleidae (annelida: Polychaeta) associated with Atlantic deep-sea reducing habitats, with the description of Ophryotrocha fabriae, new species. Proceedings of the Biological Society of Washington, 122: 14-25.

Pfannenstiel, H.D. 1977. Experimental analysis of the "Paarkultureffekt" in the protandric polychaete, Ophryotrocha puerilis Clap. Mecz. Journal of Experimental Marine Biology and Ecology, 28: 31-40.

Pleijel, F. \& Eide, R. 1996. The phylogeny of Ophryotrocha (Dorvilleidae: Eunicida: Polychaeta). Journal of Natural History, 30: 647-659.

Prevedelli, D.; N'Siala, G.M. \& Simonini, R. 2006. Gonochorism vs. hermaphroditism: Relationship between life history and fitness in three species of Ophryotrocha (Polychaeta: Dorvilleidae) with different forms of sexuality. Journal of Animal Ecology, 75: 203-212.

Ravara, A.; Marçal, A.R.; Wiklund, H. \& Hilário, A. 2015. First account on the diversity of Ophryotrocha (Annelida, Dorvilleidae) from a mammal-fall in the deep-Atlantic Ocean with the description of three new species. Systematics and Biodiversity, 2000: 1-16.

Read, G. 2016. Dorvilleidae Chamberlin, 1919. G. Read and K. Fauchald. World Polychaeta Database [1]. Available at: http://www.marinespecies.org/ polychaeta/aphia.php? $p=$ taxdetails\&id=971 on 2015-06-02. Access in: 30/12/2019.
Rouse, G.W. \& Pleijel, F. 2001. Polychaetes. Oxford, Oxford University Press. Salvo, F.; Wiklund, H.; Dufour, S.C.; Hamoutene, D.; Pohle, G. \& Worsaae, K. 2014. A new annelid species from whalebones in Greenland and aquaculture sites in Newfoundland: Ophryotrocha cyclops, sp. nov. (Eunicida: Dorvilleidae). Zootaxa, 3887: 555-568.

Schleicherova, D.; Lorenzi, M.C.; Sella, G. \& Michiels, N.K. 2010. Gender expression and group size: a test in a hermaphroditic and a gonochoric congeneric species of Ophryotrocha (Polychaeta). The Journal of Experimental Biology, 213: 1586-1590.

Sella, G. 1985. Reciprocal egg trading and brood care in a hermaphroditic polychaete worm. Animal Behaviour, 33: 938-944.

Sella, G. 1991. Evolution of Biparental Care in the hermaphroditic Polychaete Worm Ophryotrocha. Evolution, 45: 63-68.

Sella, G. 2006. Sexual strategies and mating systems. In: Rouse, G. \& Pleijel F. (Eds). Reproductive biology and phylogeny of Annelida. Enfield, Science Publishers. p. 77-92.

Sella, G. \& Lorenzi, M. 2000. Partner fidelity and egg reciprocation in the simultaneously hermaphroditic polychaete worm Ophryotrocha diadema. Behavioral Ecology, 11, 260-264.

Sella, G.; Premoli, M.C. \& Turri, F. 1997. Egg trading in the simultaneously hermaphroditic polychaete worm Ophryotrocha gracilis (Huth). Behavior and Ecolology, 8: 83-86.

Taboada, S.; Wiklund, H.; Glover, A.G.; Dahlgren, T.G.; Cristobo, J. \& Avila, C. 2013. Two new Antarctic Ophryotrocha (Annelida: Dorvilleidae) described from shallow-water whale bones. Polar Biology, 36: 1031-1045.

Wiklund, H.; Altamira, I.V.; Glover, A.G.; Smith, C.R.; Baco, A.R. \& Dahlgren, T.G. 2012. Systematics and biodiversity of Ophryotrocha (Annelida, Dorvilleidae) with descriptions of six new species from deep-sea whalefall and wood-fall habitats in the north-east Pacific. Systematics and Biodiversity, 10: 243-259.

Wiklund, H.; Glover, A.G. \& Dahlgren, T.G. 2009. Three new species of Ophryotrocha (Annelida: Dorvilleidae) from a whale fall in the Northeast Atlantic. Zootaxa, 2228: 43-56. 